\title{
Quantum dynamics of N-methylacetamide studied by the vibrational configuration interaction method
}

\author{
Hiroshi Fujisaki ${ }^{\text {a,b,* }}$, Kiyoshi Yagi ${ }^{\text {c,d }}$, Kimihiko Hirao ${ }^{c, d}$, and \\ John E. Straub ${ }^{\mathrm{a}, \mathrm{e}}$

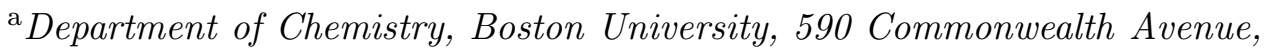 \\ Boston, Massachusetts 02215, USA \\ ${ }^{\mathrm{b}}$ Institute of Physical and Theoretical Chemistry, J.W. Goethe University, \\ Max-von-Laue-Str. 7, 60438 Frankfurt am Main, Germany \\ ${ }^{\mathrm{c}}$ Department of Applied Chemistry, School of Engineering, The University of \\ Tokyo, Hongo 7-3-1, Bunkyo-ku, Tokyo 113-8656, Japan \\ dCREST, Japan Science and Technology Agency, Saitama 332-0012, Japan \\ e Department of Chemistry and Biochemistry, Montana State University, \\ Bozeman, Montana 59717, USA
}

\begin{abstract}
Vibrational energy transfer of the amide I mode of N-methylacetamide (NMA) is studied theoretically using the vibrational configuration interaction method. A quartic force field of NMA is constructed at the B3LYP/6-31G+(d) level of theory and its accuarcy is checked by comparing the resulting anharmonic frequencies with available theoretical and experimental values. Quantum dynamics calculations for the amide I mode excitation clarify the dominant energy transfer pathways, which sensitively depend on the anharmonic couplings among vibrational modes. A ratio of the anharmonic coupling to the frequency mismatch is employed to predict and interpret the dominant energy flow pathways.
\end{abstract}

Key words: Vibrational configuration interaction (VCI) method, Vibrational energy relaxation (VER), N-methylacetamide (NMA), Quartic Force Field (QFF)

\footnotetext{
* Corresponding author: fujisaki@theochem.uni-frankfurt.de
} 


\section{Introduction}

The dynamics of proteins involves high frequency vibrational modes that behave quantum mechanically even at room temperature $(T \simeq 300 \mathrm{~K}$ corresponds to $\simeq 200 \mathrm{~cm}^{-1}$ ). One such class of vibrations is the amide I mode, centered around $1650 \mathrm{~cm}^{-1}$ and prominent in IR experiments due to its large oscillator strength. The amide I modes have been intensively studied by 2D-IR spectroscopy, which has provided new insights into their anharmonic couplings to the other vibrational modes and their intrinsic mode anharmonicities $[1,2,3]$. Theoretical studies have also been stimulated to characterize the details of the vibrational states $[4,5,6,7]$, and to probe the origin of the inhomogeneous lineshape broadening of the amide I mode $[5,8,9,10]$.

In this study, we focus on the vibrational energy relaxation (VER) process of the amide I mode. Recent pump-probe experiments $[11,12]$ have found that the VER of the amide I mode occurs on a a sub-picosecond timescale after its excitation. Nguyen and Stock [13] have studied this phenomenon using molecular dynamics and instantaneous normal mode analysis. Their results were found to be in qualitative agreement with the experimental results. Nevertheless a more precise quantum mechanical description of the VER process is desirable. Two of the authors [14] have recently proposed a time-dependent perturbation theory to describe VER, and applied the formula to a small peptide-like molecule, N-methylacetamide (NMA), in heavy water using an empirical force field. They also observed VER on a time scale $(\sim 0.5 \mathrm{ps})$ similar to that obtained experimentally, and further proposed a mechanism for the VER process.

The present study explores the VER processes of NMA more accurately by directly solving the Schrödinger equation for molecular vibrations on $a b i n i$ tio potential energy surface (PES). The NMA molecule in vacuum is examined, excluding water molecules included in the previous studies [13,14]. Quantum dynamics calculations are carried out by the vibrational configurationinteraction (VCI) method $[15,16,17,18]$. 24 vibrational modes (out of 30) are explicitly treated in the dynamics, excluding the 6 lowest-lying modes including the rotational motions of the two methyl groups. We note that while the multiconfiguration time-dependent Hartree (MCTDH) method [19] provides a more flexible framework the VCI method is sufficient for the present aim to investigate the vibrational motion of a semi-rigid molecule. 


\section{Methods}

\subsection{Quartic force field}

The PES is approximated using a fourth-order Taylor series expansion around the equilibrium geometry, which is called quartic force field (QFF), as

$$
\tilde{V}\left(\left\{Q_{i}\right\}\right)=\frac{1}{2} \sum_{k} \omega_{k}^{2} Q_{k}^{2}+\frac{1}{3 !} \sum_{k, l, m} t_{k l m} Q_{k} Q_{l} Q_{m}+\frac{1}{4 !} \sum_{k, l, m, n} u_{k l m n} Q_{k} Q_{l} Q_{m} Q_{n},(1)
$$

where $Q_{k}$ and $\omega_{k}$ denote the $k$ th normal coordinate and the associated harmonic frequency. The coefficients, $t_{k l m}$ and $u_{k l m n}$, are the third- and fourthorder derivatives of the PES. The above QFF can be recast in the form of the $n$-mode coupling representation $(n \mathrm{MR})[20,21]$ as

$$
\begin{aligned}
\tilde{V}\left(\left\{Q_{i}\right\}\right) & \simeq V\left(\left\{Q_{i}\right\}\right)=V^{1 M R}+V^{2 M R}+V^{3 M R} \\
V^{1 M R}\left(\left\{Q_{i}\right\}\right) & =\sum_{k}\left(\frac{1}{2} \omega_{k}^{2} Q_{k}^{2}+\frac{1}{3 !} t_{k k k} Q_{k}^{3}+\frac{1}{4 !} u_{k k k k} Q_{k}^{4}\right), \\
V^{2 M R}\left(\left\{Q_{i}\right\}\right) & =\sum_{k, l}\left(\frac{1}{3 !} t_{k k l} Q_{k}^{2} Q_{l}+\frac{1}{4 !} u_{k k l l} Q_{k}^{2} Q_{l}^{2}+\frac{1}{4 !} u_{k k k l} Q_{k}^{3} Q_{l}\right), \\
V^{3 M R}\left(\left\{Q_{i}\right\}\right) & =\sum_{k, l, m}\left(\frac{1}{3 !} t_{k l m} Q_{k} Q_{l} Q_{m}+\frac{1}{4 !} u_{k k l m} Q_{k}^{2} Q_{l} Q_{m}\right) .
\end{aligned}
$$

It is known that $3 \mathrm{MR}-\mathrm{QFF}$ is enough to characterize the anharmonicity of molecules studied $[20,21]$, we thus have neglected the fourth-order terms including $u_{k l m n}$ with $k \neq l \neq m \neq n$.

In addition to the above 3MR-QFF, we study two types of approximate QFF: (1) $2 \mathrm{MR}-\mathrm{QFF}$ that neglects $V^{3 M R}$ [4] and (2) partial-QFF that takes into account only the terms associated with the amide I mode, that is, $t_{k l m}=$ 0 and $u_{k k l m}=0$ if the subscripts do not include the amide I mode. Note that the previous perturbation calculation [14] also employed partial-QFF to calculate the reduced density matrix. By adding the normal mode kinetic energy $K=\sum_{k} P_{k}^{2} / 2$, we have the full approximate vibrational Hamiltonian $H=K+V\left(\left\{Q_{i}\right\}\right)$ for the system.

\subsection{Vibrational CI method}

The vibrational self-consistent field (VSCF) calculation is first carried out for the vibrational ground state to obtain the basis functions for the VCI 
calculations. The VSCF wavefunction is expressed as a direct product of onemode functions or modals as

$$
\Psi_{\mathbf{n}}^{\mathrm{VSCF}}=\prod_{i=1}^{f} \phi_{n_{i}}^{(i)}\left(Q_{i}\right)
$$

where $\mathbf{n}$ and $f$ denote the vibrational quantum numbers and the degrees of freedom, respectively. The modals are determined by

$$
\left[-\frac{1}{2} \frac{\partial^{2}}{\partial Q_{i}^{2}}+\left\langle\prod_{j \neq i} \phi_{n_{j}}^{(j)}|V| \prod_{j \neq i} \phi_{n_{j}}^{(j)}\right\rangle\right] \phi_{n_{i}}^{(i)}=\epsilon_{n_{i}}^{(i)} \phi_{n_{i}}^{(i)} .
$$

This VSCF equation is solved for the vibrational ground state $(\mathbf{n}=\mathbf{0})$, and the virtual modals constitute the VSCF configurations to be used in the VCI calculations. The VCI wavefunction is expressed as a linear combination of VSCF configurations as

$$
\Psi_{\mathbf{n}}^{\mathrm{VCI}}=\sum_{\mathbf{m}} C_{\mathbf{m n}} \Psi_{\mathbf{m}}^{\mathrm{VSCF}}
$$

The VCI wavefunction and energy levels are obtained by diagonalization of the VCI matrix

$$
H_{\mathbf{m n}}=\left\langle\Psi_{\mathbf{m}}^{\mathrm{VSCF}}|H| \Psi_{\mathbf{n}}^{\mathrm{VSCF}}\right\rangle
$$

In this study, the modals were expanded in terms of the harmonic oscillator (HO) wavefunction. The number of $\mathrm{HO}$ wavefunctions employed were 11, 9, 7 , and 5 for $\left\{\phi^{(7)}, \phi^{(8)}\right\},\left\{\phi^{(9)}-\phi^{(12)}\right\},\left\{\phi^{(13)}-\phi^{(23)}\right\}$, and $\left\{\phi^{(24)}-\phi^{(30)}\right\}$, respectively. The mode index is labeled in the increasing order of the frequency. See Table 1. The 6 lowest-lying modes were kept frozen. The VSCF configurations were constructed by allowing the excitation up to 10 quantum numbers, and selected in the increasing order of the energy until the upper limit of the VCI space, denoted $N_{C I}$, was achieved. The VSCF/VCI calculations were carried out using the SINDO code [22] for non-rotating molecules.

\subsection{Quantum dynamics}

Once the eigenvalues $\left(\left\{E_{\mathbf{n}}\right\}\right)$ and the eigenfunctions $\left(\left\{\Psi_{\mathbf{n}}^{\mathrm{VCI}}\right\}\right)$ are obtained, it is straighforward to carry out an approximate quantum dynamics simulation 
Table 1

Calculated harmonic (H.O) and anharmonic (VCI and PT2) frequencies of NMA based on the B3LYP/6-31G+(d) level of theory. The previous cc-VSCF and VCI results and the experimental results are also listed for comparison. Units in $\mathrm{cm}^{-1}$.

\begin{tabular}{ccccccc}
\hline \hline Mode & H.O. $^{a}$ & VCI $^{b}$ & PT2 $^{b}$ & cc-VSCF $^{c}$ & VCI $^{d}$ & Exp. $^{e}$ \\
\hline \hline 7 & 623 & 619 & 614 & 636 & 633 & 619 \\
8 & 630 & 625 & 613 & 637 & 685 & 658 \\
9 & 879 & 869 & 861 & 891 & 886 & 857 \\
10 & 1001 & 983 & 982 & 1022 & 1005 & 980 \\
11 & 1066 & 1046 & 1042 & 1083 & 1061 & 1037 \\
12 & 1107 & 1093 & 1080 & 1119 & 1099 & 1089 \\
13 & 1163 & 1141 & 1126 & 1184 & 1167 & - \\
14 & 1199 & 1186 & 1146 & 1214 & 1199 & 1168 \\
15 & 1292 & 1272 & 1256 & 1283 & 1253 & 1266 \\
16 & 1421 & 1397 & 1387 & 1421 & 1388 & 1370 \\
17 & 1473 & 1448 & 1432 & 1468 & 1442 & 1419 \\
18 & 1495 & 1451 & 1481 & 1515 & 1467 & 1432 \\
19 & 1500 & 1453 & 1459 & 1557 & 1483 & 1432 \\
20 & 1507 & 1469 & 1476 & 1541 & 1481 & 1446 \\
21 & 1529 & 1495 & 1481 & 1566 & 1505 & 1472 \\
22 & 1560 & 1537 & 1505 & 1547 & 1519 & 1511 \\
23 & 1751 & 1725 & 1725 & 1751 & 1727 & 1707 \\
24 & 3059 & 3017 & 2906 & 2939 & 2940 & 2915 \\
25 & 3060 & 2996 & 2940 & 2940 & 2983 & 2958 \\
26 & 3117 & 3077 & 2963 & 2979 & 2995 & 2973 \\
27 & 3132 & 3093 & 2977 & 3014 & 3043 & 3008 \\
28 & 3136 & 3100 & 2990 & 2985 & 3025 & 2973 \\
\hline \hline & 3149 & 3077 & 2990 & 2993 & 3056 & 3008 \\
30 & 3643 & 3479 & 3466 & 3523 & 3544 & 3498 \\
\hline
\end{tabular}

${ }^{a}$ Harmonic frequencies at the B3LYP/6-31+G(d) level.

${ }^{b}$ Based on 3MR-PES at the B3LYP/6-31+G(d) level.

${ }^{c}$ Reference [4]. Based on 2MR-PES at the MP2/DZP level.

${ }^{d}$ Reference [7]. Based on partial-3MR-PES at the MP2/aug-cc-pVTZ level.

e Reference [24]. 


$$
|\Psi(t)\rangle=\sum_{\mathbf{n}}\left\langle\Psi_{\mathbf{n}}^{\mathrm{VCI}} \mid \Psi(0)\right\rangle e^{-i E_{\mathbf{n}} t / \hbar}\left|\Psi_{\mathbf{n}}^{\mathrm{VCI}}\right\rangle
$$

If we take the initial state to be the VSCF configuration, $\Psi(0)=\Psi_{\mathbf{i}}^{\mathrm{VSCF}}$, then the overlap integral between $\Psi(t)$ and $\Psi_{\mathbf{j}}^{\mathrm{VSCF}}$ is calculated as

$$
O_{\mathbf{j}}(t) \equiv\left\langle\Psi_{\mathbf{j}}^{\mathrm{VSCF}} \mid \Psi(t)\right\rangle=\sum_{\mathbf{n}} C_{\mathbf{j} \mathbf{n}} C_{\mathbf{n} \mathbf{i}} e^{-i E_{\mathbf{n}} t / \hbar}
$$

The absolute square of $O_{\mathbf{j}}(t)$ gives the probability of $\mathbf{j}$ at time $t, P_{\mathbf{j}}(t)=$ $\left|O_{\mathbf{j}}(t)\right|^{2}$. Dynamics calculations were carried out with the initial VSCF configurations corresponding to the fundamental and first overtone of the amide I mode. We note that we also examined different initial states which are the superposition of states near the amide I mode fundamental or overtone, but the results do not severely depend on how to prepare the initial states [23].

\section{Results}

\subsection{Anharmonic frequency calculations of NMA: Accuracy of the PES}

Here we shall examine the accuracy of the PES derived from B3LYP/6$31+\mathrm{G}(\mathrm{d})$ employing anharmonic frequency calculations because the vibrational quantum dynamics sensitively depends on anharmonicity of a system. We compare our result with the previous theoretical values obtained by ccVSCF method [4] and very recently by VCI method [7] using MULTIMODE [17] as well as with the experimental values [24]. We have also computed the fundamental frequencies of NMA by the second-order perturbation theory (PT2) [25,26] using Gaussian03 [27]. Table 1 shows the resulting harmonic (H.O.) and anharmonic frequencies (VCI and PT2) based on the B3LYP/6$31+\mathrm{G}(\mathrm{d})$ level of theory, together with the previous cc-VSCF results based on the MP2/DZP [4] and the VCI results based on the MP2/aug-cc-pVTZ [7]. The experimental results are taken from infrared spectra of NMA in nitrogen matrix [24]. Although the experiment was not in the gas phase, we expect that the nitrogen matrix should affect minimally for vibrational frequencies, and we regard these correct values. As is well known, the harmonic frequencies overestimate the correct frequencies but the anharmonic frequencies are closer to the latter. A close inspection shows that the PT2 result is the most accurate but marginally and the other three methods are almost comparable except for six CH stretching modes $\left(\sim 3000 \mathrm{~cm}^{-1}\right)$, where our result seems to be crudest. However, we are interested in the amide I mode, whose frequency is around $1700 \mathrm{~cm}^{-1}$, and the VER pathways from the amide I mode are usually toward lower frequency modes, in this paper we accept this level of accuracy. 


\subsection{Vibrational quantum dynamics of NMA-D: Fermi resonance}

To compare with the previous dynamics calculation using a force field [14] and time-resolved experiments [11,12], we deuterated NMA into NMA-D $\left(\mathrm{CH}_{3}\right.$ ND-CO- $\mathrm{CH}_{3}$ ). We show the VER dynamics of NMA-D calculated by VCI method in Fig. 1. We consider two initial states: $v=1$ (fundamental) and $v=$ 2 (overtone) excitation of the amide I mode in the VSCF base. In both cases, the initial "decay" appears to occur on a sub picosecond timescale, which is in accord with the previous studies $[12,14]$. However, the long-time dynamics (not shown) are quasi-periodic with resonant energy "transfer" between the amide I mode and the other modes. In order to induce irreversible decay, it is essential to include more bath modes in the form of solvent molecules so that energy can dissipate [14]. A vibrationally excited molecule with sufficient quanta can decay irreversibly even in a vacuum [28]. The present result indicates that the intramolecular energy "transfer" process is responsible for the initial fast "decay" ( $\leq 1 \mathrm{ps})$.
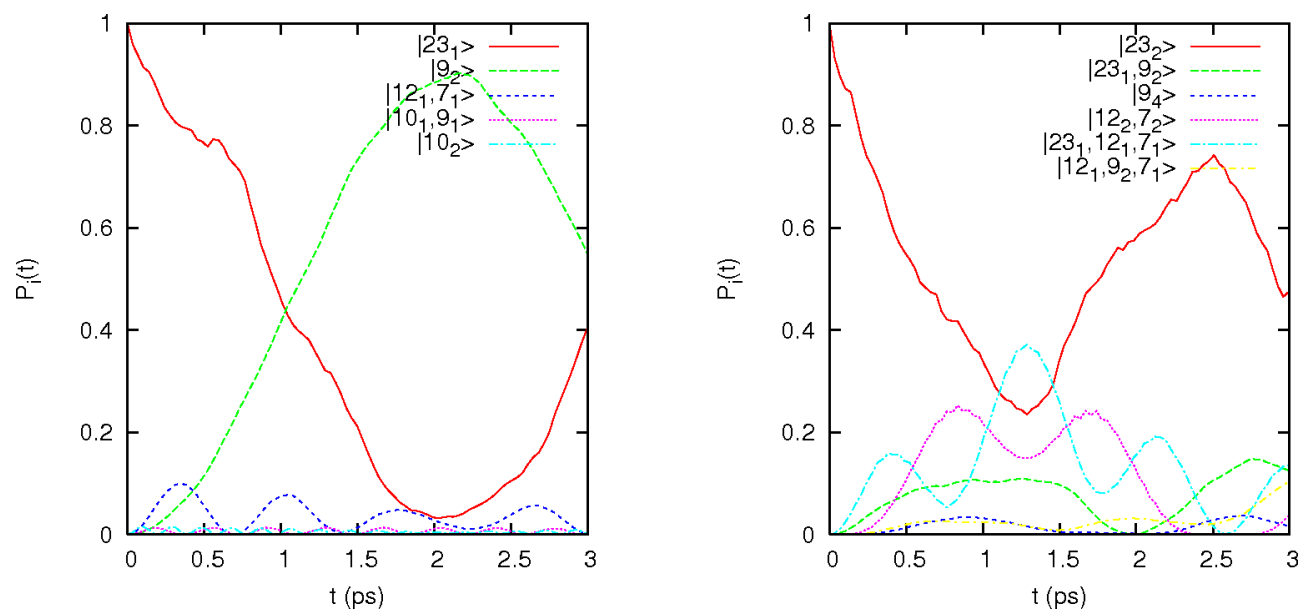

Fig. 1. Quantum population dynamics of the vibrational excited states, $v=1$ (left) and $v=2$ (right) of the amide I mode, on 3MR-QFF derived from B3LYP/6-31G+(d) method. $N_{C I}$ is set to 6000 . The dominant VER pathways are also summarized in Table 2 .

The dominant VER pathways are described in Table 2, and the resonant bath modes are depicted in Fig. 2. We interpret these dominant pathways using the following Fermi resonance parameter [29]

$$
\eta \equiv\left|\frac{\langle i|\Delta V| f\rangle}{\Delta E}\right|
$$

where $|i\rangle$ and $|f\rangle$ are the initial and final harmonic states, $\Delta V=V-$ $\sum_{k} \omega_{k}^{2} Q_{k}^{2} / 2$ is the anharmonic potential energy, and $\Delta E$ is the energy difference between $|i\rangle$ and $|f\rangle$. For example, $\eta$ for a transition between $|i\rangle=\left|S_{1}\right\rangle$ 
Table 2

The dominant VER pathways in NMA-D and the corresponding Fermi resonance parameters when we excite the fundamental of the amide I mode. $\left|m_{n}\right\rangle$ denotes the $n$th excited states of the $m$ th mode, and all other modes are on the vibrational ground state. The 23rd mode is the amide I mode in this paper. The result is derived from the B3LYP/6-31G+(d)/3MR-PES.

\begin{tabular}{cc}
\hline \hline VER pathway & Fermi resonance parameter \\
\hline \hline$\left|23_{1}\right\rangle \rightarrow\left|9_{2}\right\rangle$ & 0.082 \\
$\left|23_{1}\right\rangle \rightarrow\left|12_{1} 7_{1}\right\rangle$ & 0.024 \\
$\left|23_{1}\right\rangle \rightarrow\left|10_{2}\right\rangle$ & 0.014 \\
$\left|23_{1}\right\rangle \rightarrow\left|10_{1} 9_{1}\right\rangle$ & 0.013 \\
\hline \hline
\end{tabular}
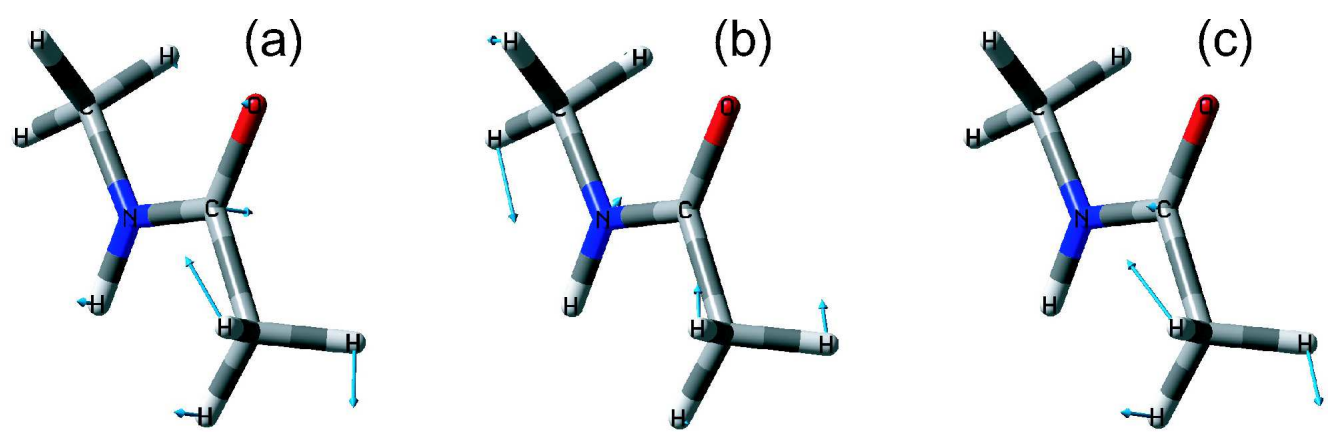

Fig. 2. Normal mode vectors of the resonant bath modes for the amide I mode in deuterated NMA. (a) 7th $\left(619 \mathrm{~cm}^{-1}\right)$, (b) 9 th $\left(869 \mathrm{~cm}^{-1}\right)$, and (c) 12th (1066 $\mathrm{cm}^{-1}$ ) mode.

(the system mode is singly excited) and $|f\rangle=\left|k_{1} l_{1}\right\rangle$ (two bath modes are singly excited) is evaluated as

$$
\eta=\left|\frac{\left\langle S_{1}\left|2 t_{S k k} Q_{S} Q_{k} Q_{l}\right| k_{1} l_{1}\right\rangle}{6 \hbar\left(\omega_{S}-\omega_{k}-\omega_{l}\right)}\right|=\left|\frac{t_{S k l}}{3 \hbar\left(\omega_{S}-\omega_{k}-\omega_{l}\right)}\right| \sqrt{\frac{\hbar}{2 \omega_{S}}} \sqrt{\frac{\hbar}{2 \omega_{k}}} \sqrt{\frac{\hbar}{2 \omega_{l}}} .
$$

Note that the Fermi resonance parameter was a key ingredient of the timedependent perturbation theory in describing the reduced density matrix for the amide I mode [14]. Similar analysis has been applied to protein dynamics of myoglobin in terms of classical mechanics [30].

Table 2 also shows that the dominant VER pathways are well characterized by the above parameters for the $v=1$ excitation. Figure 3 shows that other minor pathways take on values of $\eta$ that are smaller than $\sim 0.01$ and that the frequency matching condition is important to predict the Fermi resonance parameter. It is interesting to note that the dominant pathways (Fig. 2) are similar to those suggested in [11]. In the case of $v=2$ excitation (Fig. 1, 
right), it is difficult to assign such a parameter because the relaxation processes can accompany higher order processes, but the dominant pathways such as $\left|23_{2}\right\rangle \rightarrow\left|23_{1}, 12_{1}, 9_{1}\right\rangle$ and $\left|23_{2}\right\rangle \rightarrow\left|9_{4}\right\rangle$ are the frequency matching ones. We conclude that the Fermi resonance parameter or frequency matching condition is very important to characterize the VER pathways, and its consequence is discussed below.

\subsection{Discussions}

From these observations, we conclude that a relatively small number of resonant bath modes plays an essential role in the quantum dynamics. Simplification of the PES or a reduction in the number of modes, including the resonant bath modes, can result in an inaccurate estimate of the dynamics. This is illustrated in Fig. 4: the 3MR-QFF and partial-QFF results agree rather well up to $0.2(0.5)$ ps for $v=1(v=2)$, while 2MR-QFF results deviate from the other two at the initial stage. This is because the $2 \mathrm{MR}$ representation misses resonances mediated through the three mode interactions. It is interesting that the vibrational frequencies can be calculated accurately using $2 \mathrm{MR}-\mathrm{QFF}$; the fundamental (first overtone) of the amide I mode is obtained as 1713 (3414), and $1716(3421) \mathrm{cm}^{-1}$ by the 2MR- and 3MR-QFF, respectively (the difference is $0.2 \%$ ). However, the VER processes sensitively depend on the $3 \mathrm{MR}$ terms.

We showed that our PES is accurate enough (Sec. 3.1), which is in accord with experiment [24] and comparable to other theoretical methods [27,4,7]. However, we note that the VER dynamics are much more sensitive to accuracy than the anharmonic frequency calculations. For example, Gerber's group did not report any strong resonant interaction between the amide I mode and other modes using MP2/DZP level of theory [4]. This is likely due to the level of theory for the PES (MP2/DZP), but another possibility is due to the 2MRPES in their calculations. As we showed above, 3MR-PES and 2MR-PES can provide similar anharmonic frequencies, but some resonance conditions should be missed in the latter, affecting the dynamics calculations. Importantly, 2DIR spectroscopy can directly detect anharmonic coupling in a molecule $[1,2,3]$, and such data may be utilized to select an appropriate level of methods for a particular molecule.

\section{Concluding remarks}

Using the VCI method, we investigated the quantum dynamics of deuterated N-methylacetamide in vacuum. We demonstrated the applicability of the 

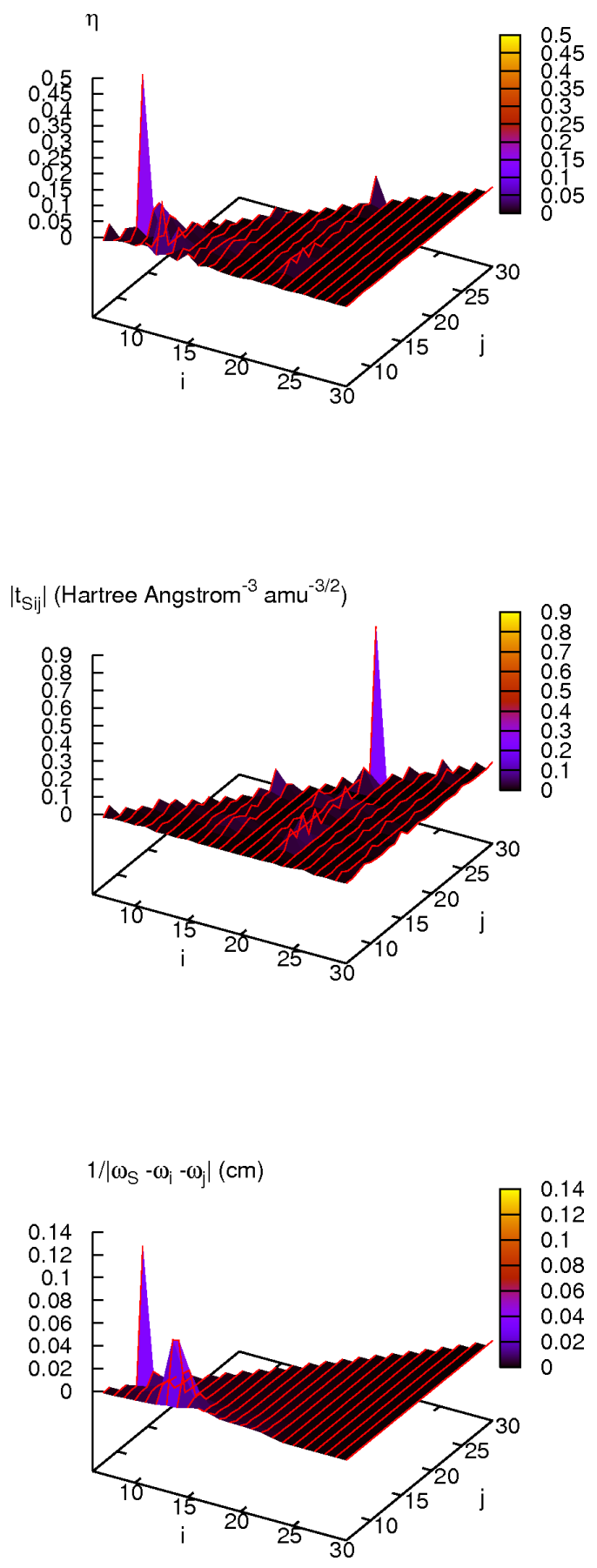

Fig. 3. Top: The Fermi resonance parameter as a function of the mode index $(i, j)$. Middle: The 3rd order coupling strength $\left|t_{\text {Sij }}\right|$ as a function of the mode index $(i, j)$. Bottom: The inverse of the frequency mismatch $1 /\left|\omega_{S}-\omega_{i}-\omega_{j}\right|$ as a function of the mode index $(i, j)$.

method and were able to gain insight into the fundamental nature of VER in the molecule, relevant to the interpretation of IR and 2D-IR spectroscopy used as a probe of protein dynamics. The accuracy of the PES employed (B3LYP $/ 6-31+\mathrm{G}(\mathrm{d}))$ was checked by the anharmonic frequency calculations 

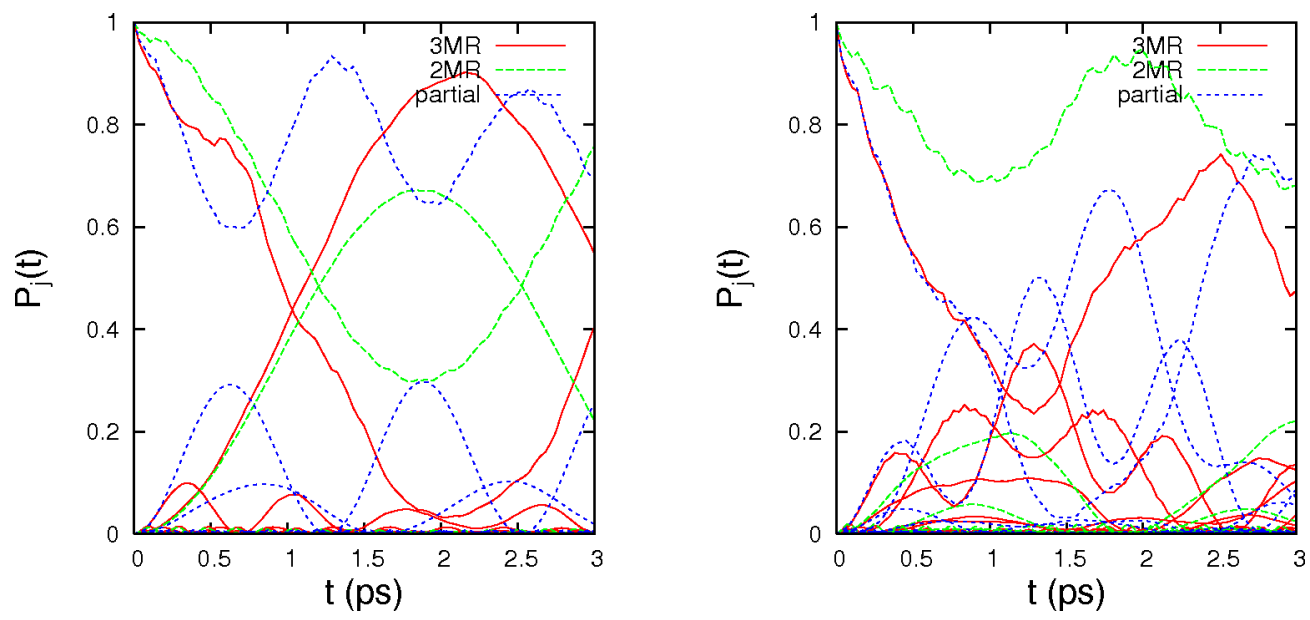

Fig. 4. Quantum population dynamics of the vibrational excited states, $v=1$ (left) and $v=2$ (right) of the amide I mode, on 3MR-QFF, 2MR-QFF, and partial-QFF, derived from B3LYP/6-31G+(d) method. $N_{C I}$ is set to 6000 .

and by comparing with experiment and other theoretical methods. We clarified the energy flow pathways from the $v=1$ and 2 excitations of the amide I mode, and interpreted our results using Fermi resonance parameters, which represent the effective coupling strength betweem vibrational modes. This approach will be extended to condensed phase systems by invoking QM/MM methods $[31,32]$ or multiresolution methods $[6,33,34]$ to deal with dephasing problems and to simulate 2D-IR signals [35].

\section{Acknowledgments}

We are grateful to E. Geva, G. Stock, D.M. Leitner, J.M. Bowman, and Y. Zhang for useful discussions. We also thank the National Science Foundation (CHE-0316551) and Boston University's Center for Computer Science for generous support to our research. JES is grateful to Montana State University for generous support and hospitality.

\section{References}

[1] M.T. Zanni, R.M. Hochstrasser, Curr. Opin. Struc. Biol. 11 (2001) 516.

[2] J.P. Wang, R.M. Hochstrasser, J. Phys. Chem. B 110 (2006) 3798.

[3] L.P. DeFlores, Z. Ganim, S.F. Ackley, H.S. Chung, A. Tokmakoff, J. Phys. Chem. B 110 (2006) 18973.

[4] S.K. Gregurick, G.M. Chaban, R.B. Gerber, J. Phys. Chem. A 106, 8696 (2002).

[5] T. Hayashi, T. la Cour Jansen, W. Zhuang, S. Mukamel, J. Phys. Chem. A 109 (2005) 64. 
[6] M. Bounouar, Ch. Scheurer, Chem. Phys. 323, 87 (2006).

[7] A.L. Kaledin, J.M. Bowman, J. Phys. Chem. A (in press).

[8] S. Ham, S. Hahn, C. Lee, M. Cho, J. Phys. Chem. B 109 (2005) 11789.

[9] J. R. Schmidt, S. A. Corcelli, J. L. Skinner, J. Chem. Phys. 121 (2004) 8887.

[10] R.D. Gorbunov, P.H. Nguyen, M. Kobus, G. Stock, J. Chem. Phys. 126, 054509 (2007).

[11] P. Hamm, M.H. Lim, R.M. Hochstrasser, J. Phys. Chem. B 102 (1998) 6123.

[12] M.T. Zanni, M.C. Asplund, R.M. Hochstrasser, J. Chem. Phys. 114 (2001) 4579.

[13] P.H. Nguyen, G. Stock, J. Chem. Phys. 119 (2003) 11350.

[14] H. Fujisaki, Y. Zhang, J.E. Straub, J. Chem. Phys. 124 (2006) 144910.

[15] J.M. Bowman, J. Chem. Phys. 68 (1978) 608.

[16] R.B. Gerber, M.A. Ratner, Chem. Phys. Lett. 68 (1979) 195.

[17] S. Carter, J.M. Bowman, N.C. Handy, Theor. Chem. Acc. 100 (1998) 191.

[18] J.M. Bowman, S. Carter, X. Huang, Int. Rev. Phys. Chem. 22 (2003) 533.

[19] M.H. Beck, J. Jäckle, G.A. Worth, H.-D. Meyer, Phys. Rep. 324 (2000) 1.

[20] K. Yagi, T. Taketsugu, K. Hirao, M.S. Gordon, J. Chem. Phys. 113 (2000) 1005.

[21] K. Yagi, K. Hirao, T. Taketsugu, M.W. Schmidt, M.S. Gordon, J. Chem. Phys. $121(2004) 1383$.

[22] K. Yagi, SINDO Version 1.3 (2006).

[23] H. Fujisaki, K. Yagi, K. Hirao, J.E. Straub, unpublished.

[24] S. Ataka, H. Takeuchi, M. Tasumi, J. Mol. Struct. 113 (1984) 147.

[25] V. Barone, J. Chem. Phys. 122 (2005) 014108.

[26] J. F. Gaw, A. Willets, W. H. Green, N. C. Handy, in: J. M. Bowman (Ed.), Advances in Molecular Vibrations and Collision Dynamics, JAI, Greenwich, CT, 1990.

[27] M.J. Frisch et al. (2004) Gaussian 03, Revision C.02, Gaussian, Inc., Wallingford CT.

[28] M. Gruebele, P.G. Wolynes, Acc. Chem. Res. 37 (2004) 261.

[29] M. Cremeens, H. Fujisaki, Y. Zhang, J. Zimmermann, L.B. Sagle, S. Matsuda, P.E. Dawson, J.E. Straub, F.E.Romesberg, J. Am. Chem. Soc. 128 (2006) 6028.

[30] K. Moritsugu, O. Miyashita, A. Kidera, Phys. Rev. Lett. 85 (2000) 3970. 
[31] S.Z. Lin, J.R. Schmidt, S. Corcelli, C.P. Lawrence, J.L. Skinner, J. Chem. Phys. 124 (2006) 204110.

[32] S. Hirata, M. Valiev, M. Dupuis, S.S. Xantheas, S. Sugiki, H. Sekino, Mol. Phys. 103 (2005) 2255.

[33] G. Rauhut, J. Chem. Phys. 121 (2004) 9313.

[34] K. Yagi, S. Hirata, K. Hirao, Theo. Chem. Acc., in press.

[35] Y. Tanimura, J. Phys. Soc. Jpn. 75 (2006) 082001. 